\title{
A Characterization of the Ligon-Schaaf Regularization Map
}

\author{
R.H. CUSHMAN \\ Universiteit Utrecht \\ AND \\ J.J. DUISTERMAAT \\ Universiteit Utrecht
}

\begin{abstract}
First, we give an explicit description of all the mappings from the phace space of the Kepler problem to the phase space of the geodesics on the sphere, which transform the constants of motion of the Kepler problem to the angular momentum. Second, among these we describe those mappings which in addition send Kepler solutions to parametrized geodesics. Third, we describe those mappings which in addition are canonical transformations of the respective phase space. Finally we prove that among these the Ligon-Schaaf map is the unique one which maps the collison orbits to the geodesics which pass through the north pole. In this way we also give a new proof that the Ligon-Schaaf map has all the properties described above.
\end{abstract}

\section{Introduction}

In order to state our characterization we describe the Kepler problem in $n$-dimensional space. As in Carathéodory [1] and Moser [7] the generalization to arbitrary dimension can be made with almost no change in the standard formulas.

We use the standard inner product $\langle x, y\rangle$ of $x, y \in \mathbf{R}^{n}$ in order to identify $x \in \mathbf{R}^{n}$ with the linear form $y \mapsto\langle x, y\rangle$ on $\mathbf{R}^{n}$. In this way we identify the phase space $P$ Tthe cotangent bundle of $\mathbf{R}^{n} \backslash\{0\}$ Wwith the set of $(q, p)$ such that $q \in \mathbf{R}^{n} \Gamma q \neq 0 \Gamma$ and $p \in \mathbf{R}^{n}$. After suitable rescaling $\Gamma$ including the multiplication of the time variable with a constant factor $\Gamma$ the equations of motion of the Kepler problem take the form

$$
\begin{aligned}
& \dot{q}=p, \\
& \dot{p}=-|q|^{-3} q,
\end{aligned}
$$

Communications on Pure and Applied Mathematics, Vol. 000, 0001-0015 (0000)

(C) 0000 John Wiley \& Sons, Inc.

CCC 0010-3640/97/000 
where we have used the notation $|q|=\langle q, q\rangle^{1 / 2}$ for the Euclidean norm of $q \in \mathbf{R}^{n}$. The right hand side of (1.1) is a Hamiltonian vector field $X_{H}$ defined by the Hamiltonian function

$$
H(q, p)=\frac{1}{2}|p|^{2}-|q|^{-1}, \quad(q, p) \in P,
$$

which is the total energy of the system.

The components $\mu_{i j}(q, p):=q_{i} p_{j}-p_{i} q_{j}$ of the angular momentum

$$
\mu(q, p):=q \wedge p
$$

are constants of motion; this follows from the rotational invariance of $X_{H}$. Additional constants of motion are the components of the eccentricity vector

(1.4) $\left.\varepsilon(q, p):=|q|^{-1} q-|p|^{2} q+\langle q, p\rangle p=|q|^{-1} q+p\right\lrcorner \mu, \quad \mu=q \wedge p$.

Here we have freely identified vectors with linear forms Tfor instance $p\lrcorner \mu$ is the interior product of the vector $p$ with the two-form $\mu$. The linear form $p\lrcorner \mu$ in turn is viewed as a vector in $\mathbf{R}^{n}$. For $n=3 \Gamma$ the components of $\varepsilon$ were described explicitly as constants of motion for the first time by Laplace [5ГPartie 1ГLivre IITChap. III]. We use the notation of Hamilton [3] Twho observed that the norm of $\varepsilon$ is equal to the eccentricity $e$ of the orbit.

Györgyi [2] seems to have been the first to observe that the components of the eccentricity vector satisfy the Poisson bracket relations

$$
\begin{aligned}
\left\{\mu_{i j}, \varepsilon_{k}\right\} & =\delta_{j k} \varepsilon_{i}-\delta_{i k} \varepsilon_{j}, \\
\left\{\varepsilon_{i}, \varepsilon_{j}\right\} & =-2 H \mu_{i j} .
\end{aligned}
$$

Here the equations (1.5) express the equivariance of $\varepsilon$ under rotations $\Gamma$ whereas (1.6) is obtained by a direct computation.

From now on we shall restrict our attention to the open subset

$$
P_{-}:=\{(q, p) \in P \mid H(q, p)<0\}
$$

of the phase space defined by the condition that the energy is negative. Because of the relations

$$
e^{2}=|\varepsilon|^{2}=1+2 H \text { and }|\mu|^{2}=|q|^{2}|p|^{2}-\langle q, p\rangle^{2},
$$

we see that the elliptical orbits $\Gamma$ that is $0 \leq e<1 \Gamma$ correspond to $H<0$ and $\mu \neq 0$. The solutions with $H<0$ and $\mu=0$ run into the origin with 
infinite speed in finite positive and negative time. These are the collision orbits.

The Poisson bracket relations (1.6) led Györgyi [2] to replace $\varepsilon$ by the rescaled eccentricity vector

$$
\eta:=\nu \varepsilon, \quad \nu:=(-2 H)^{-1 / 2}
$$

for which (1.5) $\Gamma(1.6)$ take the form

$$
\begin{aligned}
\left\{\mu_{i j}, \eta_{k}\right\} & =\delta_{j k} \eta_{i}-\delta_{i k} \eta_{j}, \\
\left\{\eta_{i}, \eta_{j}\right\} & =\mu_{i j} .
\end{aligned}
$$

The equations (1.10) and (1.11) imply that the functions $\mu_{i j}$ and $\eta_{i}$ together span a Lie algebraTtaking Poisson bracket as the Lie bracket Twhich is isomorphic to so $(n+1)$. This is seen by letting the $\eta_{i}$ play the role of $\mu_{n+1, i}$. Viewing the $\mu_{i j}$ with $1 \leq i<j \leq n+1$ together as the components of a two-form on $\mathbf{R}^{n+1}$ Twe get a mapping $J$ from $P_{-}$to the dual of the Lie algebra $\operatorname{so}(n+1)$ Twhich is the momentum mapping of an infinitesimal Hamiltonian action of $\operatorname{so}(n+1)$ on $P_{-}$. This infinitesimal action extends the standard infinitesimal rotations defined by the Lie subalgebra $\operatorname{so}(n)$.

Another bonus of the change from $\varepsilon$ to $\eta$ is that the energy is expressed in terms of $\mu$ and $\eta$ by means of the formula

$$
|J|^{2}=|\mu|^{2}+|\eta|^{2}=\nu^{2}=(-2 H)^{-1},
$$

whereas (1.8) yields a formula for $H$ in terms of $\mu$ and $\varepsilon$ only when $\mu \neq 0$.

The solutions of the Kepler system will be mapped to geodesics of the unit sphere $S$ of dimension $n$ in $\mathbf{R}^{n+1}$ on which the rotation group $\mathrm{SO}(n+1)$ acts naturally. Here we will take for the phase space the complement $T$ of the zero section in the (co)tangent bundle of $S$ Twhich can be described as the set of $(x, y) \in \mathbf{R}^{n+1} \times \mathbf{R}^{n+1}$ such that $\langle x, x\rangle=1 \Gamma$ $\langle x, y\rangle=0 \Gamma$ and $y \neq 0$. The momentum mapping of the infinitesimal Hamiltonian action of $\operatorname{so}(n+1)$ on $T$ is given by

$$
\tilde{J}:(x, y) \mapsto x \wedge y .
$$

The images of the Kepler solutions are geodesics with time rescaled by a factor which only depends on the energy. To be precise Tthe Kepler solutions are mapped to the solution curves of the Hamiltonian system defined by the Delaunay Hamiltonian $\widetilde{H}$ on $T$ Tdefined by

$$
\widetilde{H}(x, y)=-\frac{1}{2} \frac{1}{|y|^{2}}=-\frac{1}{2} \frac{1}{|\widetilde{J}|^{2}}, \quad(x, y) \in T .
$$


The components of $\tilde{J}$ are constant along the geodesics in $T$.

The Ligon-Schaaf regularization mapping is the mapping $\Phi=\Phi_{\mathrm{LS}}$ : $P_{-} \rightarrow T$ given by

$(1.15) \Phi(q, p):=((\sin \varphi) A+(\cos \varphi) B,-\nu(\cos \varphi) A+\nu(\sin \varphi) B)$,

where

$$
\begin{aligned}
& A=A(q, p):=\left(|q|^{-1} q-\langle q, p\rangle p, \nu^{-1}\langle q, p\rangle\right), \\
& B=B(q, p):=\left(\nu^{-1}|q| p,|p|^{2}|q|-1\right),
\end{aligned}
$$

and $\varphi=\varphi_{\mathrm{LS}} \Gamma$ with

$$
\varphi_{\mathrm{LS}}(q, p):=\nu^{-1}\langle q, p\rangle .
$$

Recall that $\nu=\nu(q, p)$ has been introduced in (1.9) in terms of the energy $H=H(q, p)$ Twhich in turn has been defined in (1.2). No explanation was given by Ligon and Schaaf [6] about how the mapping $\Phi$ (1.15) was found. Moreover Twe found it quite a lot of work to verify the statements following their suggestions. For instance $\Gamma$ the proof in [6] of property A) below consists of a description of a mapping $\Psi \Gamma$ followed by the statement that one can check that $\Psi$ is the inverse of $\Phi_{\mathrm{LS}}$. It took us more manipulations than we liked to prove the equations $\Psi \circ \Phi=$ id on $P_{-}$and $\Phi \circ \Psi=$ id on $T_{-}$.

Ligon and Schaaf [6] present the following properties of the mapping $\Phi_{\mathrm{LS}}$.

A) $\Phi$ is an analytic diffeomorphism from $P_{-}$onto the open subset $T_{-}$ of $T$, which consists of the $(x, y) \in T$ such that $x \neq e_{n+1}$. Here $e_{n+1}$ is the $(n+1)$-th standard basis vector in $\mathbf{R}^{n+1}$, which is the north pole of the sphere $S$.

B) $\Phi$ is a canonical transformation.

C) If $\gamma$ is a solution curve of the Kepler vector field $X_{H}$ in $P_{-}$, then $\Phi \circ \gamma$ is a solution curve of the Delaunay vector field $X_{\widetilde{H}}$ in $T$.

D) $J=\tilde{J} \circ \Phi$.

As an application $\Gamma$ Ligon and Schaaf [6] observe that $g_{P_{-}}=\Phi^{-1} \circ g_{T} \circ \Phi \Gamma$ $g \in \mathrm{SO}(n+1)$ yields a partially defined Hamiltonian action of $\mathrm{SO}(n+1)$ on $P_{-}$with momentum mapping equal to $J$. Here $g_{T}$ denotes the action of $g$ on $T$ and $g_{P_{-}}$is defined on the set of $(q, p) \in P_{-}$such that the 
$x$-component of $\Phi(q, p)$ is not equal to $g_{T}^{-1}\left(e_{n+1}\right)$. This set is equal to $P_{-}$if and only if $g \in \mathrm{SO}(n)$; otherwise it is equal to the complement of a closed analytic submanifold of codimension $n$ in $P_{-}$Twhich is actually a Lagrange submanifold.

We will take the property $D$ ) as our point of departure and prove that a mapping $\Phi$ from $P_{-}$to $T$ satisfies $\left.D\right)$ if and only if it is of the form (1.15) Twhere $A$ and $B$ are as in (1.16) and (1.17) Trespectively but $\varphi$ is an arbitrary function on $P_{-}$. Next we show that $\Phi$ satisfies $\left.D\right)$ and $\left.C\right) \Gamma$ if and only if $\varphi-\varphi_{\mathrm{LS}}$ is constant along the $X_{H \text {-solution curves } \Gamma \text { if and }}$ only if $\varphi-\varphi_{\mathrm{LS}}$ is a function of the components of $\mu$ and $\eta$. In our proof of these statements $\Gamma$ the mapping $\Phi_{\mathrm{LS}}$ of Ligon and Schaaf appears in a natural way.

Now assume that $\Phi: P_{-} \rightarrow T$ is differentiable and satisfies D). For each $h<0$ denote by $P_{h}$ and $T_{h}$ the level set for the level $h$ of the energies $H$ and $\widetilde{H}$ in $P$ and $T$ Trespectively. Let $\Phi_{h}$ denote the restriction of $\Phi$ to $P_{h}$. The fact that $J$ and $\widetilde{J}$ are momentum mappings for infinitesimal Hamiltonian actions which are transitive on $P_{h}$ and $T_{h}$ Trespectively implies that $\Phi_{h}$ pulls back the canonical symplectic form of $T$ to the restriction to $P_{h}$ of the canonical symplectic form of $P$. In this sense $\Phi$ is already quite close to being a canonical transformation. We prove that a differentiable map $\Phi: P_{-} \rightarrow T$ satisfies $\mathrm{D}$ ) and $\mathrm{B}$ ) $\Gamma$ if and only if $\varphi-\varphi_{\mathrm{LS}}$ is a function of $H$ only. Finally we show that $\Phi$ satisfies D) $\Gamma$ B) and $\Phi\left(P_{-}\right) \subset T_{-} \Gamma$ if and only if $\varphi=\varphi_{\mathrm{LS}} \Gamma$ that is $\Phi=\Phi_{\mathrm{LS}}$. In this case actually $A$ ) holds (which we verify without calculating the inverse of $\left.\Phi_{\mathrm{LS}}\right)$. The final conclusion is that the Ligon-Schaaf map is completely characterized by the properties A) - D).

Moser [7] used his regularization in order to treat solutions near collision in perturbations of the Kepler problem. He did this by applying the averaging method to the corresponding pertubation of the geodesic flow in the (co)tangent bundle of the sphere. Due to the nonuniform reparametrization of the Kepler orbits Tone has to apply the averaging method energy level by energy level $\Gamma$ see van der Meer and CushmanГ[9Гp.408]. With the Ligon-Schaaf mapping this complication does not arise. On the other hand it may be harder here to recognize $\mathrm{C}$ from its asymptotic behaviour as $q \rightarrow 0 \Gamma$ whether for a given function $f$ on $P_{-}$the function $g=f \circ \Phi_{\mathrm{LS}}^{-1}$ on $T_{-}$has an extension to $T$ which near the north pole has the smoothness which is required for the application of the averaging method. This may be an interesting problem for further investigation. 


\section{Intertwining the momentum maps}

In (1.13) we identified the (dual of the) Lie algebra $\operatorname{so}(n+1)$ with the space of two-forms on $\mathbf{R}^{n+1}$ and both $J$ and $\tilde{J}$ take their values in

$$
C:=\left\{j \in \bigwedge^{2} \mathbf{R}^{n+1} \mid \operatorname{rank} j=2\right\} .
$$

The elements of $C$ with a given norm form a single (co)adjoint orbit of which the other orbits in $C$ are the positive multiples. In this way $C$ is a cone over the manifold of oriented two-dimensional vector subspaces of $\mathbf{R}^{n+1}$.

For a mapping $\Phi: P_{-} \rightarrow T$ the condition that $\tilde{J} \circ \Phi=J$ just means that $\Phi$ maps the fibers of $J$ into the fibers of $\widetilde{J}$. It implies that the image of $J$ is contained in the image of $\tilde{J}$ Twith equality if and only if the image of $\Phi$ meets every fiber of $\widetilde{J}$. As a preparation of our description of the mappings $\Phi: P_{-} \rightarrow T$ such that $\widetilde{J} \circ \phi=J$ W we therefore investigate the images and fibers of $J$ and $\tilde{J}$.

Lemma 2.1 We have $J\left(P_{-}\right)=C=\tilde{J}(T)$. A fiber of $J$ is equal to an

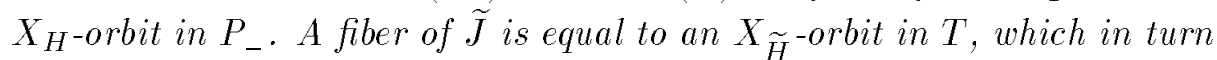
is equal to an orbit of the circle action $\alpha \rightarrow \Gamma_{\alpha}$ in $T$ defined by

$$
\Gamma_{\alpha}(x, y)=\left((\cos \alpha) x+|y|^{-1}(\sin \alpha) y,-|y|(\sin \alpha) x+(\cos \alpha) y\right)
$$

for $(x, y) \in T$ and $\alpha \in \mathbf{R} / 2 \pi \mathbf{Z}$.

Proof: We begin by proving that $J\left(P_{-}\right)=C$. In view of (1.8) with $H<0$ and (1.9) Tthe equations $\mu(q, p)=\mu$ and $\eta(q, p)=\eta$ Гwhere $\mu$ and $\eta$ are not both equal to zero and satisfy $\eta \wedge \mu=0$ Tare equivalent to the equations $\mu(q, p)=\mu$ and $\varepsilon(q, p)=\varepsilon$ Twhere $\mu$ and $\varepsilon$ are not both equal to zero and satisfy $\varepsilon \wedge \mu=0 \Gamma e=|\varepsilon| \leq 1$. Here $e=1$ if and only if $\mu=0$.

The points on a noncircular Kepler orbit which are the easiest to describe are the perihelion and the aphelion $\Gamma$ namely the points which are at the smallest and largest distance to the origin Trespectively. These are the points $(q, p)$ for which $\langle q, p\rangle=0$. Using (1.8) we get

$$
|p|^{2}=|q|^{-2}|\mu|^{2}, \quad \text { where }|\mu|^{2}=\nu^{2}\left(1-e^{2}\right) .
$$

From (1.4) we obtain

$$
\left(|q|^{-1}-|q|^{-2}|\mu|^{2}\right) q=\varepsilon \text { and } 1-|q|^{-1}|\mu|^{2}= \pm e .
$$


The positions $q_{\alpha}$ of the aphelion and $q_{\pi}$ of the perihelion therefore are given by

$$
q_{\alpha}=\nu^{2}(1+e) e^{-1} \varepsilon, \quad q_{\pi}=-\nu^{2}(1-e) e^{-1} \varepsilon .
$$

Taking the interior product of (1.3) with $\varepsilon \Gamma$ we find that the respective velocities $p_{\alpha}$ and $p_{\pi}$ of the aphelion and the perihelion are determined by the equations

$$
\left.\varepsilon\lrcorner \mu=\nu^{2}(1+e) e p_{\alpha} \text { and } \varepsilon\right\lrcorner \mu=-\nu^{2}(1-e) e p_{\pi},
$$

respectively. Conversely we find that $\mu(q, p)=\mu$ Tand $\varepsilon(q, p)=\varepsilon$ when $(q, p)=\left(q_{\alpha}, p_{\alpha}\right)$ or $(q, p)=\left(q_{\pi}, p_{\pi}\right)$ are given by $(2.3)$ and $(2.4)$. Equation (2.3) holds for the aphelia under the sole condition that $\varepsilon \neq 0$; whereas for the perihelia we also have to require that $0 \leq e<1 \Gamma$ that is $\Gamma \mu \neq 0$. In other wordsTon the set where $\varepsilon \neq 0$ Tthe aphelia define a cross-section for the mapping $J$.

The $(q, p)$ with $\varepsilon(q, p)=0$ and $\mu(q, p)=\mu \neq 0$ are given by $q=\nu^{2} v \Gamma$ $\left.p=\nu^{-2} v\right\lrcorner \mu$ Twhere $v$ is an arbitrary unit vector in $\mathbf{R}^{n}$. (These solutions also arise as the limits of the above aphelia and perihelia.) This completes the proof that $J\left(P_{-}\right)=C$.

For the description of the fibers of $J \Gamma$ we start with the case that $\mu \neq 0$. From (1.3) we see that $q$ and $p$ form a basis in the two-dimensional orthogonal complement $N$ of the kernel of $\mu$. For a given $q \Gamma$ the vector $p \in N$ is uniquely determined from $p\lrcorner \mu=|q|^{-1} q-\varepsilon \Gamma$ see (1.4). Taking inner product with $q$ in (1.4) and using (1.8) we obtain $|q|+|\mu|^{2}=\langle q, \varepsilon\rangle \Gamma$ which is an equation for $q$ which describes the Kepler ellipse in $N$. Thus when $\mu \neq 0$ Teach fiber of $J$ is equal to a Kepler orbit in phase space.

Every two-form in $\mathbf{R}^{n+1}$ of rank two can be written as $x \wedge y$ where $x$ and $y$ are linearly independent vectors in $\mathbf{R}^{n+1}$ Twhich subsequently can be chosen such that $|x|=1$ and $\langle x, y\rangle=0$. This shows that $\tilde{J}(T)=C$. The fiber of $\tilde{J}$ consists of the $(u, v) \in T$ such that $u \wedge v=x \wedge y$. This means that there exist real numbers $a, b, c, d$ such that $u=a x+b y \Gamma$ $v=c x+d y$. The conditions $\langle u, u\rangle=1 \Gamma\langle u, v\rangle=0$ and $u \wedge v=x \wedge y$ are equivalent to $a^{2}+b^{2}|y|^{2}=1 \Gamma a c+b d|y|^{2}=0$ and $a d-b c=1 \Gamma$ respectively. These hold if and only if there exists an angle $\alpha$ such that $a=\cos \alpha \Gamma b=|y|^{-1} \sin \alpha \Gamma c=-|y| \sin \alpha \Gamma d=\cos \alpha$.

The fact that $J\left(P_{-}\right)=\tilde{J}(T)$ is an encouragement to look for mappings $\Phi: P_{-} \rightarrow T$ which satisfy $J=\tilde{J} \circ \Phi$. If $\varphi$ is an arbitrary $\mathbf{R} / 2 \pi \mathbf{Z}$-valued function on $P_{-} \Gamma$ then we will write $\Phi_{\varphi}$ for the mapping $\Phi: P_{-} \rightarrow T$ as defined in (1.15) Twhere $A$ and $B$ are as in (1.16) and (1.17) Trespectively. 
A comparison of (1.15) and (2.2) shows that

$$
\Phi_{\varphi}(q, p)=\Gamma_{\varphi(q, p)}\left(\Phi_{0}(q, p)\right), \quad(q, p) \in P_{-},
$$

where $\Gamma$ is given by $(2.2)$

Proposition 2.2 A mapping $\Phi$ from $P_{-}$to $T$ satisfies $J=\tilde{J} \circ \Phi$ if and only if there exists an $\mathbf{R} / 2 \pi \mathrm{Z}$-valued function $\varphi$ on $P_{-}$, such that $\Phi=\Phi_{\varphi}$.

Proof: Write $x=\left(\widetilde{x}, x_{n+1}\right) \Gamma y=\left(\widetilde{y}, y_{n+1}\right) \Gamma$ with $\tilde{x}, \widetilde{y} \in \mathbf{R}^{n}$ and $x_{n+1}, y_{n+1} \in \mathbf{R}$. Using this notation and the definition of $J$ we see that $\widetilde{J}(x, y)=J(q, p)$ is equivalent to

$$
\begin{aligned}
\tilde{x} \wedge \widetilde{y} & =\mu=q \wedge p \\
x_{n+1} \tilde{y}-y_{n+1} \tilde{x} & =\eta=M q+N p,
\end{aligned}
$$

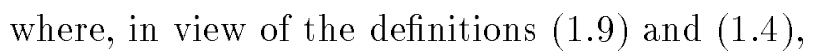

$$
M=\nu\left(|q|^{-1}-|p|^{2}\right) \quad \text { and } \quad N=\nu\langle q, p\rangle .
$$

Suppose that $\mu \neq 0$. Then (2.6) implies that there exist unique real-valued functions $a, b, c, d$ such that

$$
\widetilde{x}=a q+b p \quad \text { and } \quad \tilde{y}=c q+d p .
$$

Because $q \wedge p=\tilde{x} \wedge \tilde{y}=(a d-b c)(q \wedge p)$ Twe find that

$$
a d-b c=1 \text {. }
$$

Substituting (2.9) into (2.7) and using the linear independence of $q$ and $p$ gives a set of linear equations for $x_{4}$ and $y_{4}$ which using (2.10) may be solved to give

$$
x_{n+1}=a N-b M \text { and } y_{n+1}=c N-d M .
$$

The equations $|x|^{2}=1$ and $\langle x, y\rangle=0$ for $(x, y) \in T$ become

$$
|\widetilde{x}|^{2}+x_{n+1}^{2}=1 \text { and }\langle\widetilde{x}, \widetilde{y}\rangle+x_{n+1} y_{n+1}=0,
$$

respectively. Substituting the expressions (2.9) for $\widetilde{x}$ and $\widetilde{y}$ and the expressions (2.11) for $x_{n+1}$ and $y_{n+1}$ into (2.12) gives

$$
\begin{aligned}
& 1=a^{2}|q|^{2}+\left(\nu\langle q, p\rangle a+\nu|q|^{-1} b\right)^{2} \\
& 0=a c\left(|q|^{2}+\nu^{2}\langle q, p\rangle^{2}\right)+(a d+b c) \nu^{2}\langle q, p\rangle+b d \nu^{2}|q|^{-2}
\end{aligned}
$$


Here we have used the identities

$$
\left\{\begin{aligned}
|q|^{2}+N^{2} & =|q|^{2}+\nu^{2}\langle q, p\rangle^{2}, \\
\langle q, p\rangle-M N & =\nu^{2}|q|^{-1}\langle q, p\rangle, \\
|p|^{2}+M^{2} & =\nu^{2}|q|^{-2}
\end{aligned}\right.
$$

which follow from the definition (2.8) of $M$ and $N$ and the identity $|p|^{2}-$ $|q|^{-1}=|q|^{-1}-\nu^{-2} \Gamma$ (which is a consequence of the definition (1.9) of $\nu$ ). Multiplying (2.13) by $c$ and (2.14) by $-a$ Tadding the results gives

$$
c=-a \nu^{2}|q|^{-1}\langle q, p\rangle-b \nu^{2}|q|^{-2}
$$

after using (2.10). Similarly we obtain that

$$
d=a\left(|q|^{2}+\nu^{2}\langle q, p\rangle^{2}\right)+b \nu^{2}|q|^{-1}\langle q, p\rangle .
$$

A solution of $(2.13) \Gamma(2.15)$ and (2.16) is given by

$$
a=0, \quad b=\nu^{-1}|q|, \quad c=-\nu|q|^{-1}, \text { and } d=\nu\langle q, p\rangle .
$$

These functions clearly satisfy $(2.10) \Gamma(2.13)$ and (2.14). A comparison with (1.15) shows that $(x, y)=\Phi_{0}(q, p)$ if and only if $(2.9)$ and (2.11) hold with $a, b, c, d$ as in (2.17). This means that we have checked that $J=\widetilde{J} \circ \Phi_{0}$ on the subset of $P_{-}$where $\mu \neq 0$. Because this subset is dense in $P_{-}$and $\Phi_{0}: P_{-} \rightarrow T$ is analytic $\Gamma$ by continuity we find that $J=\widetilde{J} \circ \Phi_{0}$ on $P_{-}$.

The statement of the theorem now follows from (2.5) and the description of the fibers of $\tilde{J}$ in Lemma 2.1 .

\section{Intertwining the Kepler and Delaunay flows}

As preparation of our description of the mappings $\Phi: P_{-} \rightarrow T$ such that $\widetilde{J} \circ \Phi=J$ and $\Phi$ sends every $X_{H \text {-solution curve to an } X_{\tilde{H}^{-}} \text {-solution }}$ curve we determine the derivative of the vector valued functions $A$ and $B$ C defined in (1.16) and (1.17) Talong the solution curves of the Kepler problem.

Lemma $3.1 \quad X_{H} A=\nu^{-1}|q|^{-1} B$ and $X_{H} B=-\nu^{-1}|q|^{-1} A$.

Proof: Proposition 2.2 and Lemma 2.1 imply that $\Phi_{0}=(B,-\nu A)$

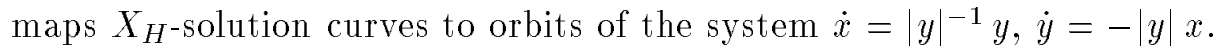


Using the fact that $|A|=|B|=1$ Tit follows that there exists a real-valued function $\lambda$ on $P_{-}$such that

$$
X_{H} B=-\lambda A \text { and }-\nu X_{H} A=\lambda(-\nu B) .
$$

Each of these equations imply that $\lambda$ is analytic $\Gamma$ because $A \Gamma B$ and $X_{H}$ are analytic.

In order to determine $\lambda$ we compute the derivative of the last coordinate of (1.16) using (1.1) as follows.

$$
X_{H} A_{n+1}=\nu^{-1} X_{H}\langle q, p\rangle=\nu^{-1}\left(|p|^{2}-|q|^{-1}\right)=\nu^{-1}|q|^{-1} B_{n+1},
$$

see (1.17). Consequently $\lambda=\nu^{-1}|q|^{-1}$ on the dense subset of $P_{-}$where $B_{n+1} \neq 0$. By continuity the same equation must hold on $P_{-}$.

In the proof of the following proposition $\Gamma$ the Ligon-Schaaf angle function $\varphi_{\mathrm{LS}}=\nu^{-1}\langle q, p\rangle$ makes its appearance in a natural way.

Proposition 3.2 The mapping $\Phi_{\varphi}$ (2.5) sends every $X_{H}$-solution curve to an $X_{\widetilde{H}^{-}}$-solution curve, if and only if $\varphi-\varphi_{\mathrm{LS}}$ is constant along the $X_{H^{-}}$ solution curves.

Proof: The condition that $\Phi_{\varphi}$ sends every $X_{H}$-solution curve to an $X_{\tilde{H}^{-}}$-solution curve implies that the function $\Phi$ is differentiable along the $X_{\tilde{H}}$-solution curves. For the moment we make no further hypotheses on $\varphi$.

In view of Proposition 2.2 and Lemma 2.1 and the fact that $X_{\widetilde{H}}$ spans the tangent space of the fibers of $\tilde{J}$ Twe find that there exists a uniquely determined real-valued function $\lambda$ on $P_{-}$Tsuch that $X_{H} \Phi_{\varphi}=\lambda X_{\tilde{H}}$. From (1.15) and Lemma 3.1 we see that if we write $\Phi_{\varphi}(q, p)=(x, y)$ where $x$ and $y$ are regarded as functions on $P_{-}$Tthen

$$
\begin{aligned}
X_{H} x= & (\cos \varphi)\left(X_{H} \varphi\right) A+(\sin \varphi) \nu^{-1}|q|^{-1} B-(\sin \varphi)\left(X_{H} \varphi\right) B \\
& \quad-(\cos \varphi) \nu^{-1}|q|^{-1} A \\
= & \nu^{-1}\left(\nu^{-1}|q|^{-1}-X_{H} \varphi\right) y .
\end{aligned}
$$

On the other hand the $X_{\widetilde{H}^{-}}$solution curves satisfy $\dot{x}=|y|^{-4} y$. Furthermore $\Gamma$ from (1.12) it follows that that $|y|=\nu$. Thus comparing these results we obtain

$$
\lambda=\nu^{3}\left(\nu^{-1}|q|^{-1}-X_{H} \varphi\right) .
$$


The condition that $\Phi_{\varphi}$ sends every $X_{H^{-}}$solution curve to an $X_{\widetilde{H}^{-}}$ solution curve implies that $\lambda=1$. In other words $\Gamma$

$$
\begin{aligned}
X_{H} \varphi & =\nu^{-1}|q|^{-1}-\nu^{-3}=\nu^{-1}\left(|q|^{-1}+2 H\right) \\
& =\nu^{-1}\left(|p|^{2}-|q|^{-1}\right)=X_{H} \varphi_{\mathrm{LS}},
\end{aligned}
$$

where the last equality follows from (3.1) and (1.18). In turn $X_{H} \varphi=$ $X_{H} \varphi_{\mathrm{LS}}$ if and only if $\varphi-\varphi_{\mathrm{LS}}$ is constant along the $X_{H}$-solution curves.

Corollary 3.3 The Ligon-Schaaf map $\Phi_{\mathrm{LS}}$ is bijective from $P_{-}$onto the set $T_{\text {- }}$ of the $(x, y) \in T$ such that $x \neq e_{n+1}$.

Proof: Here "orbit of a vector field $v$ in a manifold $M$ " means the orbit of the maximal $v$-solution curve in the manifold $M$. Proposition 3.2 implies that for each maximal $X_{H}$-solution curve $\gamma$ in $P_{-}$Tthe curve $\tilde{\gamma}:=\Phi_{\mathrm{LS}} \circ \gamma$ is an $X_{\tilde{H}^{-}}$-solution curve in $T$. The maximal solution curves of the Kepler problem are either periodic $\Gamma$ which corresponds to case that $\mu \neq 0$ Tor run into a collision in a finite time $\mathrm{b}$ both in the positive and in the negative time direction. In the first case the image of the $X_{H \text {-orbit }}$ $O$ in $P$ is a full orbit $X_{\tilde{H}^{-}}$orbit $\widetilde{O}$ in $T$ T but $\Phi_{\mathrm{LS}}$ could still be a multiple covering from $O$ to $\tilde{O}$. After we have shown that this cannot occur $\Gamma$ we conclude the proof by an investigation of $\Phi_{\mathrm{LS}}(\gamma(t))$ when $\gamma(t)$ runs into a collision and of the time between the collisions.

From Proposition 2.2 and Lemma 2.1 we see that $\Phi_{\mathrm{LS}}$ induces a bijection from the space of $X_{H^{-}}$orbits in $P_{-}$onto the space of $X_{\widetilde{H}^{-}}$-orbits in $T$. This latter space can be identified with the space of $X_{\widetilde{H}^{-}}^{\text {-orbits }}$ in $T_{-} \Gamma$ because each geodesic which starts with at $e_{n+1}$ leaves $e_{n+1} \Gamma$ so the corresponding $X_{\widetilde{H}}$-orbit meets $T_{-}$. What remains to be shown is that for each $X_{H}$-orbit $O$ the restriction to $O$ of $\Phi_{\mathrm{LS}}$ is a bijection from $O$ onto the corresponding $X_{\widetilde{H}^{-}}$-orbit in $T_{-}$.

An $X_{H}$-orbit $O$ in the subset $P_{-}^{0}$ of $P_{-}$where $\mu \neq 0$ is the orbit of a periodic solution $\gamma: \mathbf{R} \rightarrow P_{-}^{-}$of the Kepler problem. Because of Proposition 3.2 the curve $\tilde{\gamma}=\Phi_{\mathrm{LS}} \circ \gamma: \mathbf{R} \rightarrow T$ is an $X_{\tilde{H}^{-} \text {-solution } \Gamma}$ which is maximal since it is defined on $\mathbf{R}$. Therefore $\Phi_{\mathrm{LS}}(O)$ is equal to an $X_{\widetilde{H}^{-o r b i t}} \widetilde{O}$ in $T$ and $\Phi_{\mathrm{LS}}$ defines a $k$-fold covering from $O$ onto $\widetilde{O} \Gamma$ where the period of $\gamma$ is equal to $k$ times the period of $\widetilde{\gamma}$. Because the orbits in $P_{-}^{0}$ and $T$ form a fibration $\Gamma$ the number $k$ is the same for all orbits. For circular solutions where $\varepsilon=0$ we have $\langle q, p\rangle=0$ and $|p|^{2}=|q|^{-1}=$ constant. Thus the period of $\gamma$ is equal to $2 \pi|q|^{3 / 2}=2 \pi \nu^{3}$. 
On the other hand $X_{\widetilde{H}}$ is equal to $|y|^{-3}$ times the velocity field of the circle action (2.2). Thus the period of $\tilde{\gamma}$ is equal to $2 \pi|y|^{3}$. This is equal to the period of $\gamma$ because $|y|=\nu$ by (1.12). Hence $k=1$. In passing we note that we have verified that every solution in $P_{-}^{0}$ has period $2 \pi \nu^{3} \Gamma$ which is a classically know fact.

It remains to investigate the $X_{H^{-} \text {solutions }} \gamma$ in $P_{-}$for which $\mu=0$. These are the orbits which are mapped by $\Phi_{\mathrm{LS}}$ to points $(x, y)$ such that $\widetilde{x} \wedge \widetilde{y}=0$. In other words they are precisely the points on the "vertical" geodesics Tthat is Tthe geodesics which pass through the north (and south) pole. If we approximate $\gamma$ by a solution $\hat{\gamma}$ in $P_{-}^{0} \Gamma$ for instance by taking the aphelion of $\hat{\gamma}$ close to the aphelion of $\gamma$ Tthen we see that the perihelion of $\hat{\gamma}$ approaches the orgin. The velocity of $\hat{\gamma}$ is large there. Thus the time which $\hat{\gamma}$ spends near the origin tends to zero. Conseqently the time interval $[0, T]$ on which $\gamma$ is defined is approximated by a period $\left[0,2 \pi \hat{\nu}^{3}\right]$ of $\hat{\gamma}$. Therefore $T=2 \pi \nu^{3}$.

This implies that $\Phi_{\mathrm{LS}}$ maps $O$ bijectively onto the complement of a single point of the $X_{\widetilde{H}^{\text {-orbit }}}$ in $T$ and the missing point is the limit point of $\Phi_{\mathrm{LS}}(\gamma(t))$ as the position $q(t)$ of $\gamma(t)$ runs to the origin. From $|p|^{2}-2|q|^{-1}=2 H$ we find that $|p| \rightarrow \infty$ and $|p|^{2}|q| \rightarrow 2$. Hence $|p||q| \rightarrow 0$ and therefore $\varphi_{\mathrm{LS}} \rightarrow 0$. Consequently the $x$-coordinate of $\Phi_{\mathrm{LS}}(\gamma(t))$ converges to $e_{n+1}$ Tsee $(1.15)$.

\section{Canonical transformations}

Let $\omega$ and $\widetilde{\omega}$ denote the canonical symplectic form in $P_{-}$and $T$ Trespectively. In Proposition 4.3 below we describe the differentiable angle functions $\varphi$ on $P_{-}$Tfor which the mapping $\Phi=\Phi_{\varphi}(2.5)$ is a canonical transformation from $P_{-}$to $T$ Tthat is $\Gamma$ for which $\Phi^{*} \widetilde{\omega}=\omega$.

Lemma 4.1 If $\Phi=\Phi_{\mathrm{LS}}$, then $\Phi^{*} \widetilde{\omega}=\omega$.

Proof: Let $N$ denote the hypersurface in $P_{-}$where $\langle q, p\rangle=0$ Th that is the hypersurface of perihelia and aphelia Tin other words the hypersurface where $\varphi_{\mathrm{LS}}=0$ (1.18). Then the restriction of $\Phi_{\mathrm{LS}}$ to $N$ is equal to the restriction of $\Phi_{0}=(B,-\nu A)$ to $N$. We denote this mapping by $\Phi_{N}$. We also write $\iota_{N}: N \rightarrow P_{-}$for the embedding of $N$ into $P_{-}$.

From (1.16) and (1.17) we see that $-\nu A(q, p)=\left(-\nu|q|^{-1} q, 0\right)$ on $N$. Thus

$$
\Phi_{N}^{*}(\langle y, d x\rangle)=\iota_{N}^{*}\langle q, \mathrm{~d} p\rangle,
$$

because the the interior product of $-\nu|q|^{-1} q$ with $\mathrm{d}\left(\nu^{-1}|q|\right) p$ vanishes in $N$. Taking the exterior derivative of (4.1) and using the fact that the 
exterior derivative commutes with pull-backs Twe obtain

$$
\Phi_{N}^{*} \widetilde{\omega}=\iota_{N}^{*} \omega .
$$

We now restrict our attention temporarily to the subset $P_{-}^{\prime}$ of $P_{-}$ where $\varepsilon \neq 0$. In the proof of Lemma 2.1 we have seen that the perihelia and the aphelia form analytic cross-sections for the momentum mapping $J$ the fibers of which are the $X_{H^{-}}$orbits. It follows that in $P_{-}^{\prime}$ every $X_{H^{-}}$ orbit transversely intersects $N$ (twice if $\mu \neq 0$ and only once at the aphelia if $\mu=0)$. In particular $\Gamma X_{H}(q, p) \notin \mathrm{T}_{(q, p)} N$ when $(q, p) \in N \cap P_{-}^{\prime}$.

Write $\delta=\Phi_{\mathrm{LS}}^{*} \tilde{\omega}-\omega$. Let $(q, p) \in N \cap P_{-}^{\prime}$. From (4.2) it follows that $\delta_{(q, p)}(u, v)=0$ if $u, v \in \mathrm{T}_{(q, p)} N$. So in order to show that $\delta_{(q, p)}=0 \Gamma$ it suffices to verify that $\delta_{(q, p)}(u, v)=0$ if $u=X_{H}(q, p)$ and $v \in \mathrm{T}_{(q, p)} N$. Observe that $\mathrm{T}_{(q, p)} \Phi_{\mathrm{LS}}$ maps $X_{H}(q, p)$ to $X_{\widetilde{H}}(x, y) \Gamma$ where $(x, y)=\Phi_{\mathrm{LS}}(q, p)$ (see Proposition 3.2). Since the interior product of a Hamiltonian vector field with the symplectic form is equal to the derivative of the Hamiltonian function $\Gamma$ we see that $\mathrm{T}_{(q, p)} \Phi_{\mathrm{LS}} X_{H}(q, p)=$ $X_{\widetilde{H}}(x, y)$ is equivalent to the condition $\Phi_{\mathrm{LS}}^{*} \mathrm{~d} \widetilde{H}=\mathrm{d} H$ at points of $N$. However $\Gamma(1.12)$ and (1.14) imply that $H=-\frac{1}{2}|J|^{2}$ and $\widetilde{H}=-\frac{1}{2}|\widetilde{J}|^{2}$. Thus $J=\widetilde{J} \circ \Phi_{\mathrm{LS}}$ implies that $H=\Phi_{\mathrm{LS}}^{\star} \widetilde{H}$. Hence $\mathrm{d} H=\Phi_{\mathrm{LS}}^{*} \mathrm{~d} \tilde{H}$ Teven on $P_{-}$.

Finally we use the fact that the $X_{H}$-flow $F_{t}$ and the $X_{\widetilde{H}^{-}}$flow $\widetilde{F}_{t}$ leave $\omega$ and $\tilde{\omega}$ invariant $\Gamma$ respectively. Then Proposition 3.2 yields $\Phi_{\mathrm{LS}} \circ F_{t}=$ $\widetilde{F}_{t} \circ \Phi_{\mathrm{LS}}$. Thus

$$
F_{t}^{*} \Phi_{\mathrm{LS}}^{*} \tilde{\omega}=\Phi_{\mathrm{LS}}^{*} \tilde{F}_{t}^{*} \widetilde{\omega}=\Phi_{\mathrm{LS}}^{*} \tilde{\omega},
$$

which implies that $F_{t}^{*} \delta=\delta$. It follows that $\delta=0$ at all points of $F_{t}(N)$ for every $t \in \mathbf{R}$. So $\delta=0$ in $P_{-}^{\prime}$. Because $P_{-}^{\prime}$ is dense in $P_{-} \Gamma$ we obtain $\delta=0$ on $P_{-}$.

Corollary 4.2 $\Phi_{\mathrm{LS}}$ is an analytic diffeomorphism from $P_{-}$onto $T_{-}$.

Proof: We already know from Corollary 3.3 that $\Phi_{\text {LS }}$ is bijective from $P_{-}$onto $T_{-}$. From $\Phi_{\mathrm{LS}}^{*} \widetilde{\omega}=\omega$ and the nondegeneracy of $\omega$ we find that $\mathrm{T}_{(q, p)} \Phi_{\mathrm{LS}}$ is injective for every $(q, p) \in P_{-}$. Hence $\mathrm{T}_{(q, p)} \Phi_{\mathrm{LS}}$ is bijective because $\operatorname{dim} P_{-}=\operatorname{dim} T_{-}$. The conclusion now follows by applying the global inverse mapping theorem for analytic mappings.

Proposition 4.3 A differentiable map $\Phi: P_{-} \rightarrow T$ satisfies $J=\tilde{J} \circ \Phi$ and $\omega=\Phi^{*} \widetilde{\omega}$, if and only if $\Phi=\Phi_{\varphi}$ where $\varphi-\varphi_{\mathrm{LS}}$ is a function of $H$ only. Moreover, if this is the case and $\Phi\left(P_{-}\right) \subset T_{-}$, then $\Phi=\Phi_{\mathrm{LS}}$. 
Proof: In view of Proposition 2.2 it suffices to describe those differentiable angle functions $\varphi$ on $P_{-}$for which $\omega=\Phi_{\varphi}^{*} \tilde{\omega}$. Writing $\alpha=\varphi-\varphi_{\mathrm{LS}} \Gamma$ we know that

$$
\Phi(q, p)=\Gamma_{\alpha(q, p)}\left(\Phi_{\mathrm{LS}}(q, p)\right), \quad \text { when }(q, p) \in P_{-} .
$$

Differentiating this relation in the direction of the vector $v \in \mathrm{T}_{(q, p)} P_{-} \Gamma$ we get

$$
\begin{aligned}
\mathrm{T}_{(q, p)} \Phi(v)= & \mathrm{d} \alpha_{(q, p)}(v) X_{|\widetilde{J}|}(\Phi(q, p)) \\
& +\mathrm{T}_{\Phi_{\mathrm{LS}}(q, p)} \Gamma_{\alpha(q, p)}{ }^{\circ} \mathrm{T}_{(q, p)} \Phi_{\mathrm{LS}}(v) .
\end{aligned}
$$

Here $|\widetilde{J}(x, y)|=|y|(1.14) \Gamma$ that is $\Gamma \Gamma_{t}$ is the flow of $X_{|\widetilde{J}|}$.

For every $\alpha \in \mathbf{R}$ we have $\Gamma_{\alpha}^{*} \tilde{\omega}=\tilde{\omega}$. From Lemma 4.1 we see that $\Phi_{\mathrm{LS}}^{*} \tilde{\omega}=\omega$. Therefore applying $\widetilde{\omega}_{\Phi(q, p)}$ to the pair of vectors of the form given by the second term of the right hand side of (4.3) with $v$ replaced by $v_{1}$ and $v_{2}$ T we obtain $\omega_{(q, p)}\left(v_{1}, v_{2}\right)$. Using (4.3) and the fact that $\left.X_{|\widetilde{J}|}\right\lrcorner \tilde{\omega}=\mathrm{d}|\widetilde{J}| \Gamma$ from (4.3) we find that

$$
\Phi^{*} \widetilde{\omega}=\mathrm{d} \alpha \wedge \Phi^{*} \mathrm{~d}|\widetilde{J}|+\omega .
$$

Because $\Phi^{*} \mathrm{~d}|\tilde{J}|=\mathrm{d} \Phi^{*}|\widetilde{J}|=\mathrm{d}|J| \Gamma$ we see that $\Phi^{*} \widetilde{\omega}=\omega$ if and only if $\mathrm{d} \alpha \wedge \mathrm{d}|J|=0$. This last condition is equivalent to saying that $\alpha$ is locally constant on the level sets of $|J|$ and hence on level sets of $H \Gamma$ (see (1.12)). Because the level sets of $H$ are connected $\Gamma$ this holds if and only if $\alpha$ is constant on each level set of $H$. Consequently $\alpha$ is a function of $H$.

To prove the last statement in the proposition Tassume that $\varphi=\varphi_{\mathrm{LS}}+$ $\alpha^{\circ} H \Gamma$ where $\alpha$ is an $\mathbf{R} / 2 \pi \mathbf{Z}$-valued function on $\mathbf{R}_{<0}$. If $P_{h}$ denotes the level set $H=h$ in $P_{-}$Tthen $\Phi=\Gamma_{\alpha(h)}{ }^{\circ} \Phi_{\mathrm{LS}}$ on $P_{h}$. Now $\Phi_{\mathrm{LS}}\left(P_{h}\right)$ is equal to the set of $(x, y) \in T$ such that $x \neq e_{n+1}$ and $|y|=(-2 h)^{-1 / 2}=\nu . \Gamma_{\alpha(h)}$ maps the set of $\left(e_{n+1}, y\right)$ with $|y|=\nu$ to a subset of the normal bundle of the geodesic sphere $S_{\alpha(h)}$ in $S$ around $e_{n+1}$ with geodesic distance to $e_{n+1}$ equal to $\alpha(h)$. Here $S_{\alpha(h)}$ degenerates to $\left\{e_{n+1}\right\}$ and to $\left\{-e_{n+1}\right\}$ if $\alpha(h)=0$ and $\alpha(h)=\pi$ (modulo $2 \pi) \Gamma$ respectively. It follows that $e_{n+1} \notin S_{\alpha(h)}$. Hence $\Phi\left(P_{h}\right)$ is not contained in $T_{-} \Gamma$ unless $\alpha(h)=0$ modulo $2 \pi$.

\section{Bibliography}

[1] Carathéodory, C., Über die Integration der Differentialgleichungen der Keplerschen Bewegung, Sitzungsber. d. Bayerischen Akad. d. Wiss., Mat.-naturwiss. Kl., 
1945/46. pp. 57-76 = Gesammelte Mathematische Schriften, Band 2, pp. 426-445, Beck, München, 1975.

[2] Györgyi, G., Kepler's equation, Fock variables, Bacry's generators and Dirac brackets, Nuovo Cimento, 53A, 1968, pp. 717-735.

[3] Hamilton, W.R., On the application of the method of quaternions to some dynamical questions, Proc. Roy. Irish Acad., 3 Appendix, pp. xxxvi-l, $1845=$ pp. 441-448 in: The Mathematical Papers of Sir William Rowan Hamilton, vol III: Algebra. Cambridge University Press, 1967.

[4] Kustaanheimo, P. and Stiefel, E., Perturbation theory of Kepler motion based on spinor regularization, J. f. d. reine und angew. Math., 219, 1965, pp. 204-219.

[5] Laplace, P.S., Traité de Mécanique Celeste, J.B.M. Duprat, Paris 1798. Reprint: Chelsea, New York, 1966.

[6] Ligon,T. and Schaaf, M., On the global symmetry of the classical Kepler problem, Rep. on Math. Phys., 9, 1976, pp. 281-300.

[7] Moser, J., Regularization of Kepler's problem and the averaging method on a manifold, Comm. on Pure and Appl. Math, 23, 1970, pp. 609-636.

[8] Souriau, J-M., Géometrie globale du problème à deux corps, in: Modern Devolopments in Analytical Mechanics. Proceedings IUTAM-ISIMM, ed. S. Benenti et al., suppl. to vol. 117, Atti della Accad. della Scienze Torino, Cl. Sc. Fis. Mat. Natur., 1983.

[9] van der Meer, J-C. and Cushman,R., Constrained normalization of Hamiltonian systems and perturbed Keplerian motion, ZAMP, 37, 1986, pp. 402-424.

Received date of receipt. Revised date of revision. 